\title{
Significance of Stenotrophomonas maltophilia When Detected in Sputum of Ambulatory Patients with COPD
}

Thibaud Soumagne $\mathbb{D}$

Florence Levesque

Julie Milot

Krystelle Godbout

Yves Lacasse

François Maltais (iD)

Service de pneumologie, Institut Universitaire de Cardiologie et de Pneumologie de Québec, Université Laval, Québec, QC, Canada
Correspondence: Thibaud Soumagne Service de pneumologie, Institut Universitaire de Cardiologie et de

Pneumologie de Québec, Université Laval, Québec, QC, Canada

Email thibaud_soumagne@live.fr
Introduction: Stenotrophomonas maltophilia is an emerging Gram-negative MDR bacteria. In patients with chronic obstructive pulmonary disease (COPD), it is mostly found in those with severe exacerbation of COPD requiring mechanical ventilation. The significance of $S$. maltophilia when detected in the sputum of ambulatory patients with COPD is uncertain. Objective: To access the prevalence and the risk factors of the presence of $S$. maltophilia in the sputum of ambulatory patients with COPD and to determine whether it was associated with prognosis.

Methods: All consecutive unselected ambulatory patients with GOLD 2-4 COPD were recruited between January 2017 and September 2019 from the COPD clinic of a tertiary care hospital. Presence of $S$. maltophilia was defined by a positive sputum culture for S. maltophilia. Demographics, COPD characteristics, comorbidities and known predisposing risk factors associated with $S$. maltophilia were collected from medical records.

Results: S. maltophilia was detected in the sputum of 41/393 (10\%) of study participants. Comorbidities, exacerbation, use of oral steroids and carbapenems in the previous year were risk factors for the presence of $S$. maltophilia. After adjusting on confounding factors associated with mortality including age, Charlson comorbidity index and $\mathrm{FEV}_{1}$, S. maltophilia was significantly associated with mortality (adjusted hazard ratio $2.3 ; 95 \%$ CI 1.1-4.9).

Conclusion: In the current study, we found that $10 \%$ of ambulatory patients with GOLD 2 4 COPD had S. maltophilia detected in their sputum. In addition, S. maltophilia may represent a marker of overall morbidity in patients with COPD.

Keywords: Stenotrophomonas maltophilia, pulmonary disease, chronic obstructive, risk factors, prognosis

\section{Introduction}

Bacterial colonization occurs in $30 \%$ of patients with chronic obstructive pulmonary disease (COPD) when in a stable phase and it contributes to disease progression and recurrence of acute exacerbation of COPD (AECOPD). ${ }^{1}$ Stenotrophomonas maltophilia is an emerging Gram-negative MDR bacteria that is most commonly associated with respiratory infections in humans. ${ }^{2}$ In patients with COPD, it is mostly found in those with severe AECOPD requiring mechanical ventilation and associated with worse survival. ${ }^{3}$ The significance of $S$. maltophilia when detected in the sputum of ambulatory patients with COPD is uncertain. Therefore, we aimed at investigating the prevalence and the risk factors of the presence of $S$. maltophilia in 
the sputum of ambulatory patients with COPD and to determine whether it was associated with prognosis.

\section{Methods}

This retrospective study involved all consecutive unselected ambulatory patients with GOLD 2-4 COPD recruited between January 2017 to September 2019 from the COPD clinic of a tertiary care hospital (Institut universitaire de cardiologie et de pneumologie de Québec). The study was approved by the local Research Ethics Board (CRIUCPQ, 2020-3317). Data were fully anonymized in a standardized case report form to ensure data confidentiality. As such, written consent from the patients or their guardians was exempted and this study was carried out in full compliance with the Helsinki Declaration.

Presence of $S$. maltophilia was defined by a positive sputum culture for S. maltophilia. Sputum specimens were plated and cultured on usual agar media. Colonies fitting S. maltophilia phenotypic characteristic were then isolated. Identification and antibiotic susceptibility were performed with the Vitek 2 Compact System (Biomerieux, Canada). In case of inconclusive results, colonies were identified using either Vitek MS or Bruker's MALDI/TOF mass spectrometry (Bruker, Canada).

Demographics, COPD characteristics, comorbidities and known predisposing risk factors associated with S. maltophilia were collected from medical records. We searched for potential host factors potentially associated with the detection of $S$. maltophilia using bivariate correlations that were further summarized using focused principal component analysis (FPCA, "psy" package within the $\mathrm{R}$ environment) as previously described. ${ }^{4}$ A multivariate logistic regression model with backward stepwise selection was then performed to confirm which host factors, amongst those that were identified with the FPCA, were significantly associated with the presence of $S$. maltophilia in the sputum. Finally, the impact of $S$. maltophilia sputum detection on all-cause mortality was evaluated using KaplanMeier survival analysis, Log rank test, and Cox regression. Statistical analysis was performed with $\mathrm{R}$ version 4.0.3 and RStudio version 1.4.1103 (R Foundation for Statistical Computing, Vienna, Austria).

\section{Results}

Three-hundred ninety-three patients with GOLD 2-4 COPD were included in the study. Mean age was $69.0 \pm$ 8.5 years and $57 \%$ were male. Mean $\mathrm{FEV}_{1}$ was $44.3 \pm$
$16.5 \%$ of the predicted value and $49 \%$ had GOLD D COPD (Table 1). $82 \%$ were taking triple inhaled therapy, $23 \%$ were on prophylactic azithromycin and $6 \%$ had long-term oral steroids (defined by a prednisone equivalent dose of $>7.5 \mathrm{mg} \cdot$ day $^{-1}$ for more than 3 months). The most frequent comorbidities were hypertension $(52 \%)$, diabetes $(17 \%)$, coronary artery disease (CAD) $(11 \%)$ and cancer in the previous five years $(15 \%)$. No patient was admitted to the ICU department in the 12-month period prior to data collection.

S. maltophilia was detected in the sputum of $41 / 393$ (10\%) of study participants and Pseudomonas aeruginosa was found in 55/393 patients (14\%); in 12/41 patients, $S$. maltophilia was identified during the course of a moderate AECOPD. No difference was found between patients in which S. maltophilia was detected in stable condition and during an exacerbation. In six patients, $S$. maltophilia persisted in sputum for more than one year in sputum. Susceptibility of S. maltophilia strains was of $98 \%$ for levofloxacin, $98 \%$ for minocycline, $85 \%$ for trimethoprim/sulfamethoxazole and $46 \%$ for ceftazidime.

From the available clinical data, based on the FPCA, two categories of host factors were significantly associated with the presence of $S$. maltophilia in the sputum (Figure 1). The first category was directly related to COPD and included the occurrence of moderate and/or severe exacerbations in the 12-month prior to data collection (yes or no), use of antibiotics (especially carbapenems), long-term oral steroids, inhaled corticosteroids, azithromycin and CAT score. The second category was related to comorbidities and included hypertension, cancer, cerebrovascular disease, $\mathrm{CAD}$ and chronic heart failure (CHF).

The following host factors were independently associated with $S$. maltophilia sputum detection in the multivariate analysis: $\mathrm{CAD}$ and/or CHF (OR 2.7; 95\% CI 1.3-5.7), cancer in the previous 5 years (OR 3.2; 95\% CI 1.4-7.2), long term use of oral steroids (OR 4.2; 95\% CI 1.3-12.5), the occurrence of moderate and/or severe AECOPD (OR 5.7; 95\% CI 1.9-24.7) and carbapenem use (OR 13.5; 95\% CI 4.9-39.2) in the 12-month period prior to the data collection.

Mean follow up was $25.9 \pm 11.2$ months and $25.4 \pm$ 10.6 months in patients with and without $S$. maltophilia in the sputum, respectively. During the follow-up period, 34 patients died, of these 9/41 (22\%) had S. maltophilia detected in their sputum and 25/352 (7\%) had not. The 
Table I Main Characteristics of Patients with COPD Included in the Study

\begin{tabular}{|c|c|c|c|}
\hline & Presence of S. maltophilia & Controls & p-value \\
\hline & $n=4 I$ & $n=352$ & \\
\hline \multicolumn{4}{|l|}{ Demographics } \\
\hline Age, year s & $71.0 \pm 8.2$ & $68.9 \pm 8.5$ & 0.12 \\
\hline Male & $28(68)$ & $195(55)$ & 0.11 \\
\hline Body mass index, $\mathrm{kg} / \mathrm{m} 2$ & $27.2 \pm 5.6$ & $27.2 \pm 6.3$ & 0.99 \\
\hline Current smokers, \% & $8(20)$ & $84(24)$ & 0.53 \\
\hline Cumulative smoking exposure, pack-years & $42.8 \pm 18.5$ & $41.3 \pm 19.4$ & 0.64 \\
\hline \multicolumn{4}{|l|}{ Disease severity } \\
\hline CAT score & $24.0 \pm 7.8$ & $20.9 \pm 7.4$ & 0.01 \\
\hline AECOPD in the previous year & $38(93)$ & $228(65)$ & $<0.0001$ \\
\hline Moderate exacerbations & $2.5 \pm 1.9$ & $1.4 \pm 1.6$ & $<0.0001$ \\
\hline Severe exacerbations & $0.9 \pm 1.4$ & $0.1 \pm 0.4$ & $<0.0001$ \\
\hline Number of hospital admission in the previous year & $1.1 \pm 1.7$ & $0.2 \pm 0.6$ & $<0.0001$ \\
\hline GOLD A/B/C/D & $0 / 8 / 1 / 32$ & $17 / 161 / 12 / 162$ & 0.001 \\
\hline \multicolumn{4}{|l|}{ Lung function } \\
\hline FEVI post $\mathrm{BD}, \mathrm{L}$ & $0.98 \pm 0.35$ & $1.13 \pm 0.48$ & 0.06 \\
\hline FEVI post BD, \% predicted & $39.3 \pm 14.6$ & $44.9 \pm 16.7$ & 0.10 \\
\hline GOLD stage $1 / 2 / 3 / 4$ & $0 / 10 / 20 / 11$ & $8 / 118 / 160 / 66$ & 0.44 \\
\hline \multicolumn{4}{|l|}{ Therapy } \\
\hline LAMA & $40(98)$ & $340(97)$ & 1.00 \\
\hline LABA & $39(95)$ & $342(97)$ & 0.36 \\
\hline ICS & $40(98)$ & $295(84)$ & 0.02 \\
\hline Triple therapy & $38(93)$ & $284(8 \mathrm{I})$ & 0.08 \\
\hline Long term oral steroids & $7(17)$ & $16(5)$ & 0.001 \\
\hline Macrolides & $17(42)$ & $74(21)$ & 0.003 \\
\hline Antibiotics in the previous year & $38(95)$ & $221(63)$ & $<0.0001$ \\
\hline \multicolumn{4}{|l|}{ Comorbidities } \\
\hline Charlson comorbidity index & $6.1 \pm 2.7$ & $4.6 \pm 2.1$ & $<0.0001$ \\
\hline $\mathrm{CAD}$ and/or $\mathrm{CHF}$ & $2 I(5 I)$ & $100(28)$ & 0.003 \\
\hline Hypertension & $28(68)$ & $177(50)$ & 0.03 \\
\hline Diabetes & $9(22)$ & $58(17)$ & 0.38 \\
\hline Cerebrovascular disease & $7(17)$ & $19(5)$ & 0.004 \\
\hline Cancer in the last 5 years & $13(32)$ & $47(13)$ & 0.002 \\
\hline Chronic kidney disease & I (2.5) & $4(1.1)$ & 0.53 \\
\hline Bronchiectasis & $8(20)$ & $34(10)$ & 0.06 \\
\hline
\end{tabular}

Note: Values in bold are significant $(P<0.05)$.

Abbreviations: BD, bronchodilator; CAD, coronary artery disease; CAT, COPD assessment test; CHF, chronic heart failure; ECOPD, exacerbation of COPD; FEVI, forced expiratory volume in the first second; GOLD, global initiative for chronic obstructive lung disease; ICS, inhaled corticosteroids; LABA, long-acting b2-agonist; LAMA, longacting muscarinic antagonist.

Kaplan-Meier survival curve indicated that S. maltophilia sputum detection was significantly associated with mortality in this population ( $\log$ rank test $p=0.003$ ). This association remained after adjusting on confounding factors associated with mortality including age, Charlson comorbidity index and $\mathrm{FEV}_{1}$ (adjusted hazard ratio 2.3; 95\% CI 1.1-4.9) (Figure 2).

\section{Discussion}

Studies regarding S. maltophilia in COPD are scarce and most were conducted more than 15 years ago. In these studies, prevalence of S. maltophilia ranged between $0.5 \%$ in stable COPD patients and $3 \%$ in those with severe AECOPD admitted to ICU. ${ }^{1,3}$ However, prevalence and awareness of $S$. maltophilia have increased in the last 


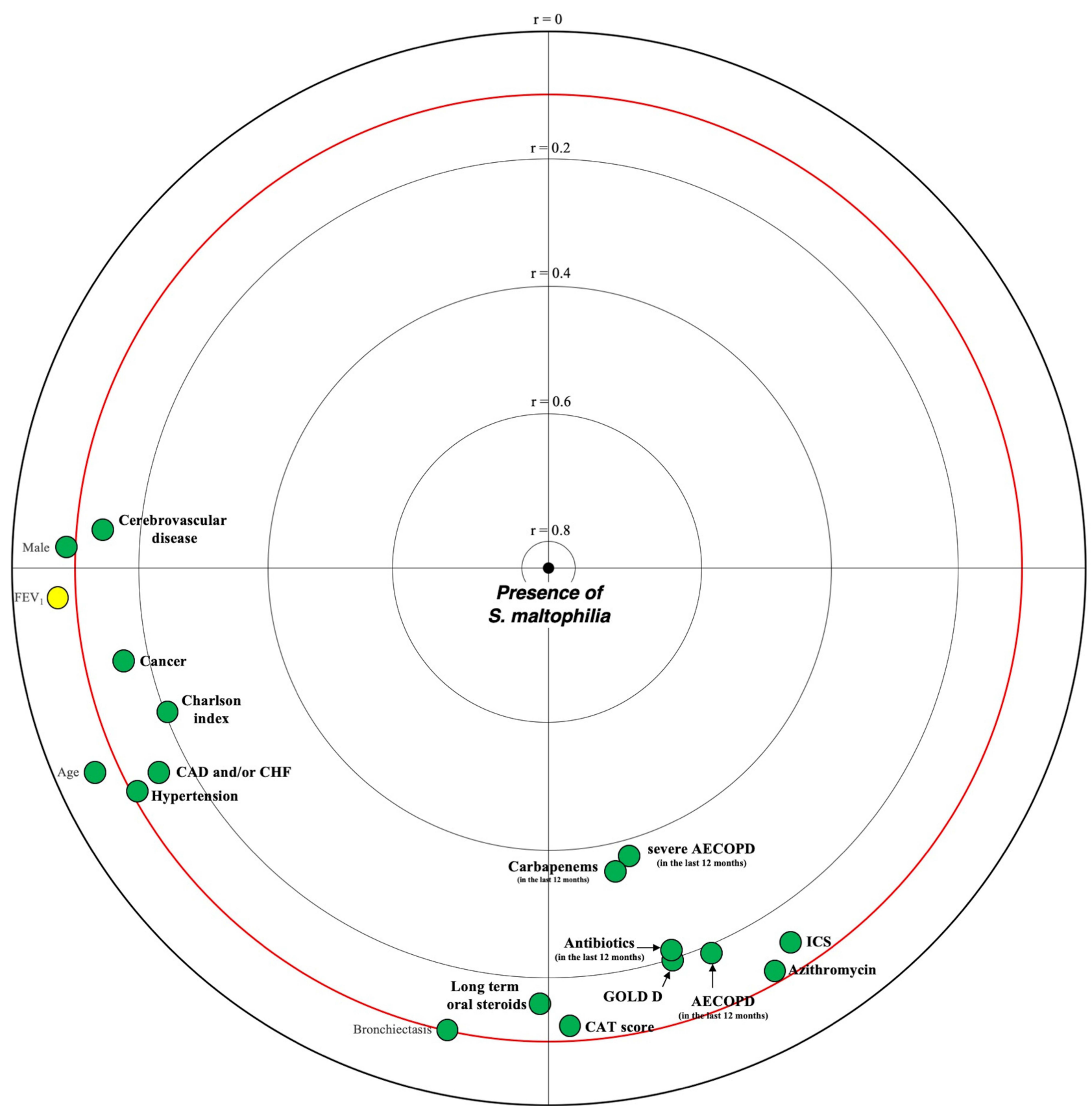

Figure I Focused principal component analysis (FPCA) for the association between presence of S. maltophilia and several host factors. FPCA is a simple graphical display of correlation structures focusing on a particular dependent variable. The display reflects primarily the correlations between the dependent variable and all other variables (covariates) and secondarily, the correlations among the covariates. The dependent variable (the presence of $S$. maltophilia in the sputum) is at the center of the diagram, and the distance from this point to a covariate faithfully represents their pairwise Spearman correlation coefficient (using ranked values of continuous variables). Green covariates are positively correlated and yellow covariates negatively correlated with the dependent variable. Covariates (in bold) inside the red circle are significantly correlated with the dependent variable (with a $p$ value $<0.05$ ). The diagram also shows relationships between covariates as follows: correlated covariates are close (for positive correlations, allowing identification of clusters) or diametrically opposite vis-a-vis the origin (for negative correlations), whereas independent covariates make a right angle with the origin.

decade and this bacteria now accounts for $1.4 \%$ of pathogens isolated from general population in Canadian hospitals. ${ }^{5}$ Furthermore, refinement in microbiological techniques, including the use of selective culture media and of MALDI-TOF mass spectrometry have improved the ability to identify and isolate of $S$. maltophilia from polymicrobial cultures. ${ }^{6,7}$ In the current study, we found a $10 \%$ prevalence of $S$. maltophilia isolation in ambulatory patients with COPD which is somewhat similar to that found in cystic fibrosis $(14 \%){ }^{8}$ 

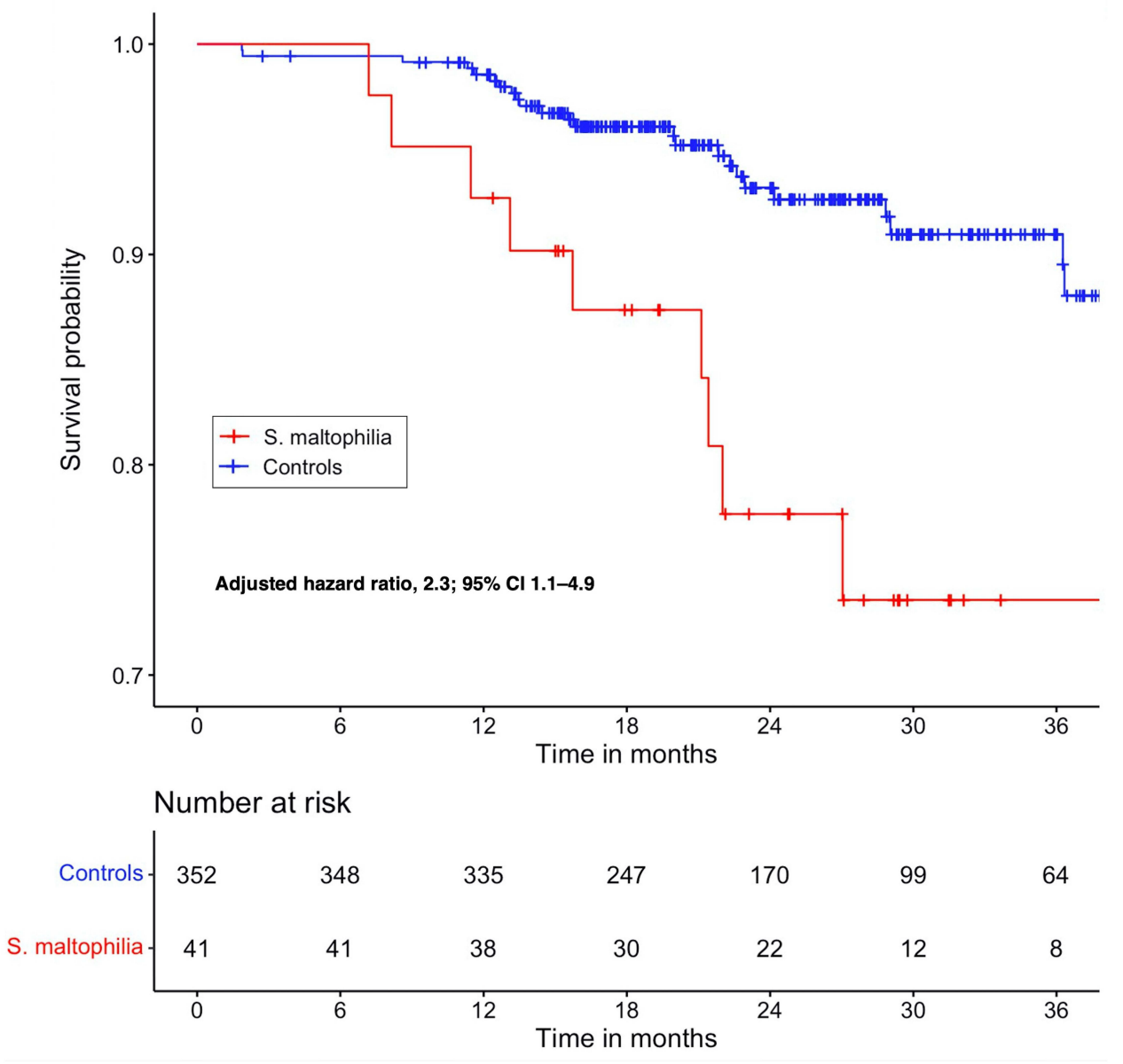

Figure 2 Kaplan-Meier survival curves over the first 36 months following sputum detection of S. maltophilia. Survival data was censored at 36 months. Mortality was adjusted for age, Charlson comorbidity index and $\mathrm{FEV}_{1}$.

Risk factors for $S$. maltophilia sputum detection in the current study were similar to those found in cancer patients, immunocompromised hosts and individuals previously exposed to wide-spectrum antibiotics. ${ }^{2,9,10}$ In addition, we found an association between $S$. maltophilia and COPD exacerbation, use of oral steroids and carbapenems which might represent targets for further preventive strategies.

As $S$. maltophilia may persist in the sputum of these patients for a long period of time, it may be difficult to ascertain the clinical significance of a positive culture result from the microbiology laboratory. ${ }^{11}$ The present data suggest that in ambulatory patients with COPD, S. maltophilia may represent a marker of overall morbidity and its clinical significance should not be overlooked by clinicians. We found that the presence of $S$. maltophilia in the sputum was associated with increased mortality even after adjustment for potential confounders such as age, Charlson comorbidity index and $\mathrm{FEV}_{1}$.
In conclusion, we found that: 1) $10 \%$ of ambulatory patients with GOLD 2-4 COPD had S. maltophilia detected in their sputum, 2) comorbidities, exacerbation, use of oral steroids and carbapenems were risk factors for the presence of S. maltophilia in the sputum, and 3) the latter may represent a marker of overall morbidity and a predictor of mortality in patients with COPD. Additional research is needed to determine the role of $S$. maltophilia in COPD.

\section{Acknowledgments}

The authors thank Dr France-Emilie Roy (Department of Microbiology, Infectious Diseases and Immunology) for her assistance.

\section{Author Contributions}

All authors made a significant contribution to the work reported, whether that is in the conception, study design, execution, acquisition of data, analysis and interpretation, or in all these areas; took part in drafting, revising or 
critically reviewing the article; gave final approval of the version to be published; have agreed on the journal to which the article has been submitted; and agree to be accountable for all aspects of the work.

\section{Disclosure}

Dr. Godbout reports grants and personal fees from AstraZeneca, Sanofi, Novartis and GSK, personal fees from Merck, Covis and TEVA, outside the submitted work. Dr. Maltais reports grants from GlaxoSmithKline, AstraZeneca, Sanofi, Novartis, Boehringer Ingelheim and Grifols, personal fees from GlaxoSmithKline, Boehringer Ingelheim, Grifols, Novartis, outside the submitted work. He also reports financial participation in Oxynov, a company which is developing an oxygen delivery system. The other authors have no conflicts of interest in this work.

\section{References}

1. Soler N, Ewig S, Torres A, Filella X, Gonzalez J, Zaubet A. Airway inflammation and bronchial microbial patterns in patients with stable chronic obstructive pulmonary disease. Eur Respir J. 1999;14 (5):1015-1022. doi:10.1183/09031936.99.14510159

2. Brooke JS. Stenotrophomonas maltophilia: an emerging global opportunistic pathogen. Clin Microbiol Rev. 2012;25(1):2-41.
3. Nseir S, Di Pompeo C, Brisson H, et al. Intensive care unit-acquired Stenotrophomonas maltophilia: incidence, risk factors, and outcome. Crit Care. 2006;10(5):R143. doi:10.1186/cc5063

4. Falissard B. Focused principal component analysis: looking at a correlation matrix with a particular interest in a given variable. J Comput Graph Stat. 1999;8(4):906-912.

5. Zhanel GG, Adam HJ, Baxter MR, et al. Antimicrobial susceptibility of 22746 pathogens from Canadian hospitals: results of the CANWARD 2007-11 Study. J Antimicrob Chemother. 2013;68 (Suppl 1):i7-22. doi:10.1093/jac/dkt022

6. Goncalves-Vidigal P, Grosse-Onnebrink J, Mellies U, Buer J, Rath PM, Steinmann J. Stenotrophomonas maltophilia in cystic fibrosis: improved detection by the use of selective agar and evaluation of antimicrobial resistance. $J$ Cyst Fibros. 2011;10(6):422-427. doi:10.1016/j.jcf.2011.06.010

7. Brooke JS. New strategies against Stenotrophomonas maltophilia: a serious worldwide intrinsically drug-resistant opportunistic pathogen. Expert Rev Anti Infect Ther. 2014;12(1):1-4. doi:10.1586/ 14787210.2014.864553

8. Canadian cystic fibrosis registry. Annual data report. 2019.

9. Toro MDD, Rodríguez-Baño J, Herrero M, et al. Clinical epidemiology of stenotrophomonas maltophilia colonization and infection: a Multicenter Study. Medicine. 2002;81(3):228-239. doi:10.1097/ 00005792-200205000-00006

10. Ding X, Wu X, Peng Y, Hu S, Nie H. Risk factors of superinfection following imipenem/cilastatin therapy in hospitalised patients with acute exacerbations of severe chronic obstructive pulmonary disease. Int J Clin Pract. 2006;60(7):806-811. doi:10.1111/j.1742-1241.2006.00829.x

11. Millar FA, Simmonds NJ, Hodson ME. Trends in pathogens colonising the respiratory tract of adult patients with cystic fibrosis, 19852005. J Cyst Fibros. 2009;8(6):386-391. doi:10.1016/j. jcf.2009.08.003

\section{Publish your work in this journal}

The International Journal of COPD is an international, peer-reviewed journal of therapeutics and pharmacology focusing on concise rapid reporting of clinical studies and reviews in COPD. Special focus is given to the pathophysiological processes underlying the disease, intervention programs, patient focused education, and self management protocols. This journal is indexed on PubMed Central, MedLine and CAS. The manuscript management system is completely online and includes a very quick and fair peer-review system, which is all easy to use. Visit http://www.dovepress.com/testimonials.php to read real quotes from published authors. 